\title{
Do Pregnancy and Parenthood Affect the Course of PCO Syndrome? Initial Results from the LIPCOS Study (Lifestyle Intervention for Patients with Polycystic Ovary Syndrome [PCOS])
}

\author{
Haben Schwangerschaft und Elternschaft Einfluss auf den Verlauf des PCO-Syndroms? \\ Erste Daten der LIPCOS-Studie (Lebensstilintervention beim Polyzystischen Ovar Syndrom [PCOS])
}

Authors

Affiliations
J. Stassek ${ }^{1}$, F. Ohnolz ${ }^{2}$, Y. Hanusch ${ }^{2}$, M. Schmidmayr ${ }^{2}$, D. Berg ${ }^{3}$, M. Kiechle ${ }^{2}$, V. R. Seifert-Klauss ${ }^{1,4}$

${ }^{1}$ Gyn. Ambulanz, Frauenklinik und Poliklinik der TU München, München

2 Frauenklinik, Klinikum rechts der Isar der Technischen Universität München, München

${ }^{3}$ Akademische Lehrpraxis der Technischen Universität München, Reproduktionsmedizin Bogenhausen, München

${ }^{4}$ Interdisziplinäres Osteoporosezentrum IOZ, Klinikum rechts der Isar, München

\section{Key words \\ infertility \\ pregnancy \\ testosterone \\ - hormones \\ - androgen \\ ovary}

\section{Schlüsselwörter}

- Infertilität

- Schwangerschaft

- Testosteron

- Hormone

- Androgen

- Ovar

Deutsche Version unter: www.thieme-connect.de/ ejournals/gebfra $\begin{array}{ll}\text { received } & 30.6 .2015 \\ \text { revised } & 21.9 .2015 \\ \text { accepted } & 21.9 .2015\end{array}$

Bibliography

DoI http://dx.doi.org/

10.1055/s-0035-1558186

Geburtsh Frauenheilk 2015; 75 :

1153-1160 @ Georg Thieme

Verlag KG Stuttgart · New York ·

ISSN 0016-5751

\section{Correspondence}

Dr. Vanadin Regina Seifert-

Klauss, MD PhD

Frauenklinik und Poliklinik

der TU München

Gyn. Ambulanz

Klinikum rechts der Isar

Ismaninger Straße 22

81675 München

vanadin.seifert-klauss@

Irz.tum.de

\section{Abstract}

\section{$\nabla$}

Introduction: The impact of pregnancy and parenthood on the long-term course of PCOS (polycystic ovary syndrome is still not known. The LIPCOS study (Lifestyle Intervention for Patients with Polycystic Ovary Syndrome [PCOS] using the example of pregnancy and parenthood) systematically investigates long-term changes in PCOS symptoms.

Method and Patients: The LIPCOS pilot study sent out a questionnaire to 403 patients who had presented with oligomenorrhea between 1991 and 2002. The prospective LIPCOS main study systematically investigated 64 women using structured interviews about lifestyle changes in the last 10 years, created a detailed hormone profile of these women and carried out vaginal ultrasound to calculate ovarian score.

Results: Ovarian volume and ovarian score were not significantly lower for women with children $(n=25)$ compared to women with PCOS who had not had children $(n=39 ; p=0.226)$. More women with children than women who did not have children currently reported a regular daily lifestyle, and the difference was statistically significant (92\% [ $n=23 / 25]$ vs. $61.5 \%$ [ $=24 / 39] ; p=0.009$ ). Ten years ago or before the birth of their first child, respectively, no such difference was found between both groups ( 52 vs. $51.3 \%$ ). Over the last 10 years, women with children were more likely to have shorter cycles compared to women without children ( $p=0.441$ ). $88 \%$ of women with children compared to $69.2 \%$ of women without children reported that currently they had a "healthy diet" $(p=0.130)$. Serum testosterone levels were slightly lower for women with children $(67.6 \%$ of the upper limits of normal ranges) compared to women without children ( $80 \%$ of the upper limits of normal ranges), but because of the small subgroup sizes the difference was not statistically significant $(\mathrm{p}=0.106)$.

\section{Zusammenfassung \\ $\nabla$}

Einleitung: Bislang ist der Einfluss von Schwangerschaft und Elternschaft auf die Langzeitentwicklung des PCO-Syndroms unbekannt. In der LIPCOS-Studie (Lebensstilintervention bei Polycystischem Ovar Syndrom [PCOS] - am Beispiel Schwangerschaft und Elternschaft) werden systematisch Langzeitveränderungen der PCOS-Symptome betrachtet.

Methoden und Patientinnen: Die LIPCOS-Pilotstudie lud 403 Patientinnen, die sich zwischen 1991 und 2002 mit Oligomenorrhö vorgestellt hatten, zur Beantwortung eines Fragebogens ein. Die prospektive LIPCOS-Hauptstudie untersuchte 64 Frauen mit PCO-Syndrom systematisch mittels strukturierten Interviews zu Lebensstilveränderungen in den letzten 10 Jahren, detailliertem Hormonprofil und vaginaler Sonografie mit Ovarscore.

Ergebnisse: Sowohl das Ovarvolumen wie auch der Ovarscore waren bei Müttern $(n=25)$ nicht signifikant geringer als bei PCO-Frauen ohne Kin$\operatorname{der}(n=39 ; p=0,226)$. Statistisch signifikant mehr Mütter als kinderlose Frauen hatten aktuell einen regelmäßigen Tagesablauf ( $92 \%$ [ $n=23 / 25]$ vs. $61,5 \%$ [n=24/39]; $\mathrm{p}=0,009$ ). Zehn Jahre zuvor bzw. vor Geburt des 1. Kindes war dies in beiden Gruppen mit 52 vs. 51,3\% nicht unterschiedlich gewesen. Mütter hatten häufiger eine Zyklusverkürzung als kinderlose Teilnehmerinnen in den letzten 10 Jahren $(\mathrm{p}=0,441)$. 88\% der Mütter vs. $69,2 \%$ der kinderlosen Teilnehmerinnen gaben aktuell „bewusste Ernährung“ an ( $p=0,130)$. Die Serum-Testosteronwerte waren etwas niedriger bei Müttern (67,6\% der oberen Normgrenze) als bei kinderlosen Frauen (80\% der oberen Normgrenze), aufgrund der kleinen Untergruppen jedoch nicht signifikant unterschiedlich $(\mathrm{p}=0,106)$. Schlussfolgerung: In der LIPCOS-Studie wird erstmals gezeigt, dass Schwangerschaft und Elternschaft einen Einfluss auf den longitudinalen 
Conclusion: The LIPCOS study shows for the first time that pregnancy and parenthood may have an impact on the long-term course of PCOS. Women with children reported shorter cycles and had lower testosterone levels compared to women without children.
Verlauf eines PCO-Syndroms haben könnten. Mütter berichteten über kürzere Zykluslängen und zeigten niedrigere Testosteronspiegel als kinderlose Frauen.

\section{Introduction}

$\nabla$

Polycystic Ovary Syndrome (PCOS) affects between 6 and 20\% of all women [1]. PCOS is characterized by anovulation (often oligomenorrhea), hyperandrogenism and polycystic ovaries on ultrasound. Different criteria are used for diagnosis, depending on the definition, which makes it more difficult to compare data. PCOS is the most common endocrine cause of infertility, menstrual cycle disorders and hirsutism in women of child-bearing age [2]. Key pathomechanisms of PCOS include hyperandrogenism and insulin resistance with compensatory hyperinsulinemia, which can develop into type 2 diabetes $[3,4]$. The etiology of PCOS remains unclear. PCOS is associated with disorders of the biosynthesis of steroid hormones which are affected by insulinlike growth factors (IGF) and IGF-binding proteins. The IGF system is therefore considered a relevant co-gonadotropic factor. Insulin resistance and compensatory hyperinsulinemia are significant accompanying features of PCOS and have been found to be present in 11 to $50 \%$ (depending on the patient population) of lean as well as obese patients with PCOS [5]. In addition to regulating the reproductive, metabolic and hyperandrogenic symptoms of PCOS by administering various drugs (oral contraceptives, antidiabetic medication, anti-androgens, etc.), lifestyle changes such as diet and exercise have increasingly been discussed in recent years as useful in the treatment of PCOS [1]. It is well known, particularly when treating obese women, that weight loss can have a positive effect on hormone disorders and metabolic changes. Despite the assumption that lifestyle changes in PCOS patients should be the first-line treatment of choice, there is little data available on this aspect. A recently published Cochrane review by Moran and colleagues [6] included only six controlled studies suitable for evaluation, which were based on the data of a total of 164 women. The observation period in these studies from the USA, Sweden and Italy was between 12 and 48 weeks. Three of the studies compared physical activity to minimal nutritional and behavioral advice or no advice. Three other studies compared combined nutritional, exercise and behavioral interventions with minimal intervention (information flyer). The studies included neither primary fertility parameters such as pregnancies, live births, or miscarriages, nor were data on ovulation or menstrual cycles reported. Lifestyle interventions resulted in improvements in overall testosterone levels, hirsutism (evaluated using the Ferriman Gallwey score) and fasting insulin levels (mean difference $-2.02 \mu \mathrm{U} / \mathrm{mL}$; $95 \% \mathrm{CI}$ : -3.28 to -0.77 ; $\mathrm{p}=0.002$ ) compared to minimal treatment. There was no evidence of lifestyle interventions affecting the body mass index, the free androgen index, or sex hormone-binding globulin (SHBG), glucose or cholesterol levels; there were also no data on quality of life, patient satisfaction or acne [6].

Overall, there are few longitudinal data on how the clinical and endocrine symptoms of PCOS change over the longer term. In particular, there is very little literature on patients' lives after becoming pregnant. The long-term clinical observation that fewer patients with PCOS ask for medical appointments for PCOS after pregnancy points to possible changes in PCOS symptoms as a result of pregnancy or becoming a parent.

This study aimed to investigate the following issues:

Do lifestyle changes due to pregnancy or parenthood affect the long-term course of PCOS, using the criteria "serum hormone levels" and "ovarian morphology"? And do psychosocial factors influence menstrual cycles in patients with PCOS?

\section{Study Participants and Methods \\ $\nabla$}

\section{Study design}

This study of the impact of lifestyle intervention on patients with polycystic ovary syndrome (PCOS) and the potential effects on the long-term course of clinical and laboratory parameters using the example of pregnancy and parenthood (LIPCOS) consists of two sub-studies:

1. A retrospective longitudinal comparative study (LIPCOS pilot study) of patients with oligomenorrhea/amenorrhea and infertility who underwent medical examination between 1991 and 2002 because of an inability to conceive. The women were requested to complete a questionnaire and also invited to participate in the LIPCOS main study.

2. A prospective longitudinal study (LIPCOS main study), which recruited participants between December 2008 and March 2011, with participants consisting of women who participated in the pilot study as well as other patients with PCOS. The main study consists of a general examination, presented in this paper, along with a prospective follow-up over a period of several years which is not the topic of this publication.

\section{Study population}

For the LIPCOS pilot study 403 patients with oligomenorrhea/ amenorrhea and/or hyperandrogenism were identified in a large infertility database and their feedback was requested using a questionnaire. They were also invited to participate in the prospective LIPCOS main study. Other patients were recruited into the prospective LIPCOS main study from the outpatient clinic of the Frauenklinik und Poliklinik of the Technical University of Munich (Director: Prof. Dr. Marion Kiechle) and the hospital Klinikum rechts der Isar in Munich and through the offices of referring physicians working in and around Munich as well as from a PCOS self-help group based in Munich.

The LIPCOS study was submitted to the ethics committee of the Technical University of Munich which evaluated it positively.

\section{Inclusion criteria}

Patients with spontaneous (not post-pill) oligomenorrhea/amenorrhea, some of whom had clinically or biochemically verified hyperandrogenism (acne, hirsutism), were included in the LIPCOS pilot study. Oligomenorrhea was the main symptom and sufficient for inclusion. The criteria for inclusion into the LIPCOS main study were specified in accordance with the Rotterdam criteria of 2003 [7]. Patients had to fulfil at least two of the following three criteria: 


\section{Anovulation}

2. Hyperandrogenism

3. Polycystic ovaries

Oligomenorrhea was defined as a menstrual cycle of $>35$ days and amenorrhea as a menstrual cycle of $>90$ days.

\section{Exclusion criteria}

Exclusion criteria for both the LIPCOS pilot study and the LIPCOS main study were the use of all medication which affects the hypothalamic-pituitary-gonadal axis such as hormonal contraceptives, estrogens or progestogens for hormone therapy, endocrine therapeutic substances to treat breast cancer or GnRH analogs for endometriosis. Women who were pregnant or breast feeding were also excluded from the study. Patients with hyperandrogenism or oligomenorrhea caused by another known endocrine disease such as androgen-producing tumor, primary hypothalamic amenorrhea or premature ovarian insufficiency or prolactinoma were also excluded from the study.

\section{Study design of the LIPCOS pilot study}

A questionnaire was sent to participating patients, and patients were requested to return the completed questionnaire. The questionnaire consisted of questions about the patient's current cycle length, the patient's cycle length 12 years ago, and the patient's medical history in pregnancy.

\section{Study design of the LIPCOS main study}

After informed written consent, a structured interview was carried out with all study participants. The interview was divided into seven subsections:

The first part ("Personal Information") asked questions about the patient's civil status, own children, desire to have children and occupation (including shift work) as well as the work-related volume of travel.

The second subsection ("Health Information") covered the patient's own medical history of PCOS. Questions were asked about the prevalence of PCOS in the patient's family, the main symptoms, the start of symptoms, and the type of prior treatments the patient had undergone for PCOS. The patient was then questioned about other co-morbidities: cardiovascular disease, abnormal cycles, amenorrhea outside pregnancy and breastfeeding, gynecological disease, gynecological surgery and other diseases/ intake of medications (especially to treat the thyroid, pituitary or adrenal gland).

The third part of the questionnaire ("Long-term Course") included open questions about significant changes in lifestyle with regard to civil status, health, nutrition, sports, the patient's occupation, environment or stress factors. Patients were also asked about serious problems they had experienced such as psychological stressors, serious illnesses, accidents or operations. The answers were initially written down freely as told and subsequently coded into different categories. The respective BMI was calculated based on the patient's information on size and weight (today vs. 5 years ago vs. 10 years ago). This part was followed by questions about the average menstrual cycle length in days (1st day of period till 1 st day of subsequent period) and the regularity of the menstrual cycle (yes/no) at the present time, 5 years ago and 10 years ago. Menstrual cycles which lasted up to 35 days were defined as eumenorrhea; a cycle length of > 35 days was termed oligomenorrhea. All reports of menstrual cycles lasting more than 91 days were classified as amenorrhea. Patients were also asked about their use of hormonal contraceptives (oral con- traceptives, hormone IUD) or other intake of hormonal preparations. Other questions focused on androgenization phenomena (acne, alopecia, hirsutism) and high serum glucose and cholesterol levels. Patients were asked to assess the following aspects of their lives 10 years ago and in the present or - where applicable - before the birth of their first child and after the birth of their first child, using the response options "applies" or "does not apply": regular daily routine, healthy diet, smoking, exercise/sports. Daily routine was defined using questions about regular sleep and meal times as well as regular activities such as regular exercise.

The fourth part of the questionnaire ("Pregnancy and Birth") consisted of questions on the means of conception (sexual intercourse without hormonal stimulation [HS], sexual intercourse with HS, insemination, in vitro fertilization [IVF]/intra-cytoplasmic sperm injection [ICSI]) and complications during pregnancy, during delivery, or postpartum. Women were also asked whether they had breastfed their children.

The fifth part ("Health and Development of the Children") addressed questions such as birth weight and marked abnormalities (e.g. congenital disease, malformations or disabilities).

The sixth part ("Life with Children") asked about changes in PCOS symptoms, menstrual cycle, weight, acne, alopecia and hirsutism (better/worse/the same, respectively) in the first two years after the birth of the child and over the longer term.

Finally, in the seventh part ("Menopause") if applicable participants were asked about their age at the last period and at the start of menopause, their medication, and any changes in PCOS symptoms since menopause.

This analysis is based primarily on the evaluation of information obtained from the questionnaire subsections 1, 2, 3 und 6. A blood sample was taken from all participants, and a vaginal ultrasound was done to calculate the ovarian score.

\section{Vaginal ultrasound and ovarian score}

Measurement of the uterus was done in 3 dimensions, the endometrium was measured in 2 dimensions, and the ovaries in $3 \mathrm{di}-$ mensions. The largest number of follicles in a single plane, the diameter of the largest follicle and the arrangement of the follicles were determined. Free fluid was documented where present along with any other findings. Patients were requested to state the time of their last period and the current day in their cycle. To quantify ovarian morphology which would allow findings to be compared with those of other studies, a functional ovarian score was calculated based on Geisthövel's classification [8]. The score incorporates different individual scores to take account of the fact that increases in ovarian size can have two quite different causes: the number of follicles or hyperplasia of the ovarian stroma.

Determination of ovarian score $(-2$ to +2$)$ was done according to the classification used in $\bullet$ Table 1. On ultrasound, points were given according to maximum ovarian diameter, number of follicles $<10 \mathrm{~mm}$ and central echogenicity of the right and the left ovary (score). The three individual scores were then added together to make the total score (ovarian score).

In this classification the \pm 0 ovary corresponds to the ovarian structure of an ovary physiologically in the early follicle phase. The $-1 /-2$ ovaries correspond to smaller ovaries such as those primarily found after menopause or secondarily in patients with severe hypothalamic amenorrhea. Ovaries with a total score of +1.5 , particularly ovaries with a score of +2 , are characteristic for hypersensitive ovaries and correspond to polycystic ovaries. 
Table 1 Ovarian score.

\begin{tabular}{|c|c|c|c|c|c|c|c|}
\hline \multirow{2}{*}{$\begin{array}{l}\text { Maximum diameter } \\
(\mathrm{mm})\end{array}$} & \multirow[t]{2}{*}{ Score } & \multirow{2}{*}{$\begin{array}{l}\text { Number of } \\
\text { follicles }<10 \mathrm{~mm}\end{array}$} & \multirow[t]{2}{*}{ Score } & \multirow[t]{2}{*}{ Central echogenicity } & \multirow[t]{2}{*}{ Score } & \multicolumn{2}{|c|}{ Ovarian score } \\
\hline & & & & & & Right & Left \\
\hline$>30 \mathrm{~mm}$ & 1 & $\geq 8$ & 0.5 & $>1 / 3$ of the overall organ & 0.5 & 2 & 2 \\
\hline$\geq 28 \mathrm{~mm}$ & 0.5 & $\geq 8$ & 0.5 & $<1 / 3$ of the overall organ & 0 & 1 & 1 \\
\hline$<28 \mathrm{~mm}-\geq 25 \mathrm{~mm}$ & 0 & $\geq 5-<8$ & 0 & $<1 / 3$ of the overall organ & 0 & & \\
\hline$<25 \mathrm{~mm}-\geq 20 \mathrm{~mm}$ & -0.5 & $\geq 3-<5$ & -0.5 & $<1 / 3$ of the overall organ & 0 & -1 & -1 \\
\hline$<20 \mathrm{~mm}$ & -1 & $<3$ & -1 & $<1 / 3$ of the overall organ & 0 & -2 & -2 \\
\hline
\end{tabular}

Ovarian volume was calculated using a simplified version of the ellipsoid formula as follows: ovarian volume $(\mathrm{ml})=$ Diameter 1 $(\mathrm{mm}) \times$ Diameter $2(\mathrm{~mm}) \times$ Diameter $3(\mathrm{~mm}) \times 0.000523$ [9]. Diameter 1 is the longitudinal length, Diameter 2 the anterior-posterior length and Diameter 3 the transverse length of the ovary. The follicle count was determined by counting and adding up all follicles in both ovaries with diameters between 2 and $10 \mathrm{~mm}$ [7].

\section{Laboratory tests}

The following values were determined using venous blood samples: CRP, complete blood cell count with blood cell morphology, LH, FSH, 17ß-estradiol, prolactin (PRL), total testosterone, thyroid-stimulating hormone (TSH), dehydroepiandrosterone sulfate (DHEAS), sex-hormone-binding globulin (SHBG). Blood samples were taken at different times in the patients' cycles, as the study participants were not taking hormone medication, and outpatient dates had to fit in with the participants' schedules.

\section{Statistical analysis}

The data were first recorded in writing on paper and later entered in tables using Microsoft Office Excel for Windows.

Data were analyzed using the programs SPSS and Microsoft Office Excel for Windows. Statistical evaluation of the data was done together with the Institute for Medical Statistics and Epidemiology (IMSE) of the Technical University of Munich (Director: Prof. K.A. Kuhn).

Continuous variables were described using the mean and standard deviation $( \pm S D)$. The Mann-Whitney U-test was used to test whether differences between two variables were significant. Categorical variables were described using absolute and relative frequencies, and Fisher's exact test was used to test for significance. The level of significance was set at $\mathrm{p}<0.05$.

\section{Results}

In the LIPCOS pilot study, participants reported that cycle length decreased from an average of 40 days to 31 days over a period of approximately 12 years. Total testosterone values measured 12 years ago were available for 133 patients. Total testosterone did not differ significantly between the 70 patients who had not been pregnant, the 23 women who had had a miscarriage, and the 21 women who had given birth. Participants with mild oligomenorrhea (>35-89 days, average duration of infertility 3.6 years) and patients with amenorrhea $>90$ days (average duration of infertility 3.1 years) had comparable rates of miscarriage.

Out of 72 patients who gave their consent and were screened for participation in the LIPCOS main study, eight (11.1\%) could not be included in the LIPCOS main study: five women only remembered that they had taken hormonal contraceptives during the interview, one woman was taking hormone therapy (HT) at the time of the study. One patient was diagnosed as not having PCOS based on the Rotterdam criteria, and one woman withdrew from the study before the start of the investigation. A total of 64 patients with a mean age of $33.6( \pm 8.5)$ years and an average current BMI of $25.2( \pm 6.5)$ remained and was included in the study. The average waist-to-hip ratio was $0.8( \pm 0.09)$, with an average waist circumference of $84( \pm 20) \mathrm{cm}$ and an average hip circumference of $104.9( \pm 19.5) \mathrm{cm}$. Mean BMI 10 years ago was calculated from the information on weight and size provided by the participants and was $22.9( \pm 4.7)$. Analysis of serum parameters obtained from the total study population using blood samples taken at some time during the woman's cycle revealed a mean LH/FSH ratio of $2.1( \pm 1.15)$ and mean $17 \beta$-estradiol levels of $108.8 \mathrm{pg} / \mathrm{ml}$ ( \pm 95.9 ; range 11.4 to 482.3 ). Mean prolactin was 11 $( \pm 5.16) \mathrm{ng} / \mathrm{ml}$, mean SHBG was $50.6( \pm 28.5) \mathrm{nmol} / \mathrm{l}$, and mean DHEAS was $2.45( \pm 1.57 \mu \mathrm{g} / \mathrm{ml})$. As different assays were used to detect testosterone and TSH over the course of time, the values are not directly comparable. The parameters are therefore reported as \% of the upper limit of the respective assay. Accordingly, mean testosterone levels were $75 \%( \pm 55.14 \%)$ of the upper limit of normal (ULN), and TSH levels were $46 \%$ ( $\pm 27.8 \%$ ) of the ULN for the respective assay.

The total study population consisted of 25 women with children and 39 women had not had children. $\triangle$ Table 1 shows the abovementioned characteristics and compares them for women with children and women without children.

The average age of women with children was 38.2 years, making them significantly older than the group of women without children (30.7 years; $\mathrm{p}<0.001$ ). Mean LH/FSH ratio and mean testosterone levels were not significantly lower for women with children; however, women with children had significantly lower prolactin levels compared to participants without children (8.3 vs. $12.9 \mathrm{ng} / \mathrm{ml} ; \mathrm{p}<0.001)$. DHEAS levels were also lower for women with children compared to women without children (1.87 vs. $2.78 \mu \mathrm{g} / \mathrm{ml} ; \mathrm{p}=0.063$ ). BMI and waist-to-hip ratio (WHR) were almost identical for both subgroups. Differences in other hormone levels were also minimal and not clinically relevant for the two subgroups with PCOS.

\section{Ovarian score}

The ovarian score for the right ovary was significantly lower in women with children than in women without children $(+0.7$ [ \pm 1.3$], \mathrm{n}=23$ vs. +1.5 [ \pm 0.7$], \mathrm{n}=35 ; \mathrm{p}=0.004)$. The ovarian score for the left ovary was not significantly different $(+0.9[ \pm 1.2]$, $\mathrm{n}=23$ vs. $+1.3[ \pm 0.9], \mathrm{n}=35 ; \mathrm{p}=0.226$ ).

Women with children had a lower ovarian volume on the right side $(10.04[ \pm 5.74] \mathrm{ml}, \mathrm{n}=21$ ) compared to women without children $(12.74[ \pm 7.09] \mathrm{ml}, \mathrm{n}=34)$ as well as on the left side (9.06 $[ \pm 6.26] \mathrm{ml}, \mathrm{n}=20$ vs. $9.96[ \pm 6.02] \mathrm{ml}, \mathrm{n}=35$ ). 


\section{Menstrual cycle history}

The menstrual cycle history of the study population showed that the mean cycle length had decreased from 53.5 to 48.7 days in the last 10 years. 0 Table 2 shows the changes in cycle length over the last 10 years for women with and without children. $44 \%$ of women with children reported a relevant decrease in the length of their menstrual cycle of $\geq 5$ days (average cycle length for all women with children decreased from 56.6 to 49.3 days); however, only $28.2 \%$ of women without children reported a relevant decrease in cycle length (average cycle length for all women with PCOS without children decreased from 52.1 to 48.3 days) $(p=0.441 ; n$. s. $)$. A decrease or increase in cycle length was only rated as a change in cycle length if the difference was $\geq 5$ days.

$52 \%$ of the women reported that their current cycle length was normal (eumenorrhea, defined as a cycle length of between 28 and $\leq 35$ days). Only $36 \%$ of women with children reported eumenorrhea in the period prior to the birth of their first child. $46.2 \%$ of women without children had a normal cycle length at the time of the current study; 10 years ago the percentage with eumenorrhea had been $1.8 \%$ lower, i.e. $44.4 \%$. At the time of the current study, $24 \%$ of women with children and $41 \%$ of women without children had oligomenorrhea; $41 \%$ of women with children and $33.3 \%$ of women without children reported oligomenorrhea in the period spanning the previous 10 years. $24 \%$ of women suffering from PCOS who had children had amenorrhea at the time of the present study; the same percentage of women who had children reported having amenorrhea (cycle length $>90$ days) before giving birth to their first child. $12.8 \%$ of women without children had amenorrhea at the time of the present study; 10 years before, $22.2 \%$ of women in the group without children had had amenorrhea. Because of the small subgroup sizes these differences were not statistically significant.

The cycle length of 7 women with children shortened from oligomenorrhea to eumenorrhea; only one of the women without children had this pattern of cycle change. Cycle length changes ranged from shortening of the cycle by 67 days to lengthening of the cycle by 61 days (both changes occurred in the last 10 years) (๑Table 3).

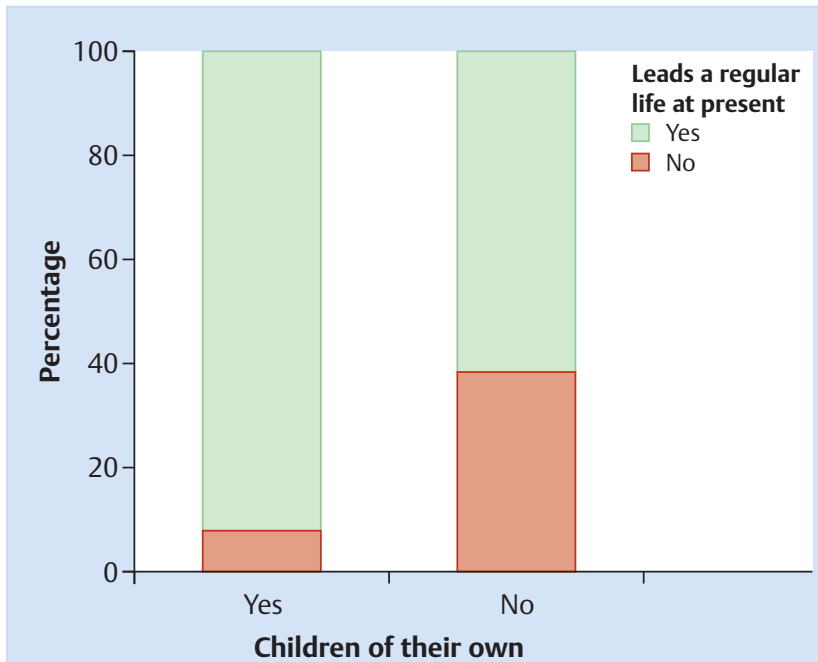

Fig. 1 Comparison of the percentage of persons who have a regular daily routine between the group of women with children $(n=25)$ and the group of women without children $(n=39)$.

\section{Stress factors and lifestyle}

When asked about their current lifestyle it became clear that significantly more women with children than women without children reported having a regular daily routine $(92 \%$ [ $=23 / 25]$ vs. $61.5 \%[n=24 / 39] ; p=0.009$ ). Ten years previously or before the birth of their first child, the same percentage of women in each group reported having a regular daily routine ( $52 \%[n=13 / 25]$ vs. $51.3 \%$ [ $n=20 / 39]$; $p>1.000$ ). O Fig. 1 compares the current percentage of participants who have a regular daily routine for the two groups with children and without children.

At the time of the interview $88 \%$ of women with PCOS who had children $(n=22 / 25)$ and $69.2 \%(n=27 / 39)$ of women with PCOS who had no children reported that they had a healthy diet $(p=0.13)$. Before the birth of their first child, $68 \%(n=17 / 25)$ of women in the group of women with children reported having a healthy diet. In the group of women without children, 56.4\%

Table 2 Comparison of parameters for women with children vs. those for women without children.

\begin{tabular}{|c|c|c|c|c|c|c|c|}
\hline & \multicolumn{7}{|c|}{ Children of their own } \\
\hline & \multicolumn{3}{|l|}{ Yes } & \multicolumn{4}{|l|}{ No } \\
\hline & Mean & \pm SD & $\mathbf{N}$ & Mean & \pm SD & $\mathbf{N}$ & $\mathbf{p}$ \\
\hline Age (years) & 38.24 & 7.25 & 25 & 30.67 & 7.98 & 39 & $<0.001$ \\
\hline Current BMI (kg/m²) & 24.88 & 4.99 & 25 & 25.33 & 7.36 & 39 & 0.693 \\
\hline BMI 10 years ago $\left(\mathrm{kg} / \mathrm{m}^{2}\right)$ & 22.72 & 5.25 & 25 & 22.97 & 4.42 & 36 & 0.606 \\
\hline Waist circumference $(\mathrm{cm})$ & 81.67 & 11.74 & 24 & 85.47 & 24.13 & 36 & 0.602 \\
\hline Hip circumference (cm) & 101.04 & 11.91 & 24 & 107.42 & 22.98 & 36 & 0.566 \\
\hline Waist-to-hip ratio & 0.82 & 0.08 & 24 & 0.79 & 0.10 & 36 & 0.122 \\
\hline CRP (mg/dl) & 0.16 & 0.16 & 25 & 0.35 & 0.47 & 35 & 0.134 \\
\hline Leukocytes (G/I) & 6.53 & 2.23 & 24 & 6.66 & 1.57 & 37 & 0.626 \\
\hline LH/FSH & 1.95 & 1.22 & 25 & 2.13 & 1.12 & 38 & 0.392 \\
\hline $17 \beta$-estradiol (pg/ml) & 114.90 & 114.86 & 25 & 104.75 & 82.44 & 38 & 0.843 \\
\hline Prolactin (ng/ml) & 8.30 & 2.91 & 25 & 12.85 & 5.58 & 36 & $<0.001$ \\
\hline Thyrotropin (\% of the upper limit) ${ }^{*}$ & 44.03 & 23.30 & 23 & 47.31 & 30.57 & 36 & 0.954 \\
\hline Testosterone (\% of the upper limit) ${ }^{*}$ & 67.60 & 59.81 & 25 & 79.96 & 52.07 & 38 & 0.106 \\
\hline DHEAS $(\mu \mathrm{g} / \mathrm{ml})$ & 1.87 & 0.92 & 22 & 2.78 & 1.77 & 38 & 0.063 \\
\hline SHBG (nmol/l) & 47.42 & 18.24 & 25 & 52.91 & 34.16 & 34 & 0.894 \\
\hline
\end{tabular}

\footnotetext{
* Values are given in this form because two different assays were used in the study population.
} 
Table 3 Comparison of cycle lengths between women with and women without children over a period of approximately 10 years. Duration of the cycle (in days) is given as mean, SD and range or as an absolute or relative frequency.

\begin{tabular}{|c|c|c|c|c|c|c|c|}
\hline & & \multicolumn{6}{|c|}{ Children of their own } \\
\hline & & \multicolumn{3}{|c|}{ Yes $(n=25)$} & \multicolumn{3}{|c|}{ No $(n=39)$} \\
\hline & & & \pm SD & Range & & $\pm S D$ & Range \\
\hline \multirow[t]{6}{*}{ Length of menstrual cycle (days) } & At present & 49.3 & 25.71 & $(20-91)$ & 48.33 & 23.84 & $\begin{array}{l}(20- \\
91)\end{array}$ \\
\hline & 10 years ago & 56.56 & 25.41 & $(28-91)$ & 52.11 & 25.21 & $\begin{array}{l}(28- \\
91)\end{array}$ \\
\hline & Shorter now than 10 years ago* & \multicolumn{3}{|c|}{$11(44 \%)$} & \multicolumn{3}{|c|}{$11(28.2 \%)$} \\
\hline & Longer now than 10 years ago* & \multicolumn{3}{|c|}{$5(20 \%)$} & \multicolumn{3}{|c|}{$9(23.1 \%)$} \\
\hline & Length remained normal** & \multicolumn{3}{|l|}{$2(8 \%)$} & \multicolumn{3}{|c|}{$8(20.5 \%)$} \\
\hline & Remained longer** & \multicolumn{3}{|c|}{$7(28 \%)$} & \multicolumn{3}{|c|}{$11(28.2 \%)$} \\
\hline
\end{tabular}

* or prior to the birth of the 1st child for women with children

** compared to 10 years ago or before the birth of the 1 st child for women with children

$(\mathrm{n}=22 / 39)$ reported that they had a healthy diet 10 years ago $(\mathrm{p}=0.435)$.

Fewer women with children $(56 \% ; n=14 / 25)$ than women without children $(61.5 \% ; n=24 / 39)$ reported that they currently did exercise on a regular basis ( $p=0.795$, n.s.). Before the birth of their first child or 10 years ago $52 \%$ of women with children $(n=13 / 25)$ and $64.1 \%$ of women without children $(n=25 / 39)$ reported that they exercised regularly $(\mathrm{p}=0.436, \mathrm{n} . \mathrm{s}$.).

At the time of the survey fewer women with children smoked than women without children $(16 \%$ [ $n=4 / 25]$ vs. $38.5 \%$ [ $n=15 /$ 39]; $\mathrm{p}=0.091$ ). Before the birth of their first child or 10 years ago, the percentage of women who smoked was approximately the same in both groups (36\% [n=9/25] vs. 38.5\% [n=15/39]; $\mathrm{p}>1.000$ ).

In the group of women without children $(\mathrm{n}=39), 28.2 \%(\mathrm{n}=11$ / 39 ) reported that their menstrual cycle had become shorter over the past 10 years. In the group of women without children who reported a shorter menstrual cycle, 90.9\% $(n=10 / 11)$ reported having a structured daily routine. Around half of the participants in the study whose menstrual cycle became longer or remained the same reported having a structured daily routine. The difference was marginally significant $(p=0.056)$.

As expected, a significantly larger percentage of women with children reported having a structured daily routine compared to the group of women without children. Women with children also had healthier diets and smoked less than women without children.

In the group of women without children who experienced a shortening of their menstrual cycle in the last 10 years, the majority $(90.9 \%, n=10 / 11)$ had a structured daily routine.

\section{Discussion}

$\nabla$

In gynecological investigations of PCOS, fertility is a very important endpoint but not a starting point for further research. The impact of weight and weight loss and of drug therapies on fertility is far better documented than the impact of changes in lifestyle, as the Cochrane meta-analysis by Moran et al. showed [5, 10].

While Legro [11] was able to demonstrate a clear correlation between fertility and BMI, the meta-analysis on the impact of lifestyle changes on fertility did not provide reliable data; most studies did not define or record cycle lengths or ovulation.
This study examines for the first time the impact of pregnancy and parenthood as lifestyle interventions on women with PCOS. There are few events which change everyday life more radically or permanently than the birth (or the adoption) of a child. These lasting lifestyle interventions resulted in significantly more women with children (92\%) having a regular daily routine compared to women without children (61\%), although previously there had been only minimal differences between the groups (52 vs. 51.3\%). Despite the more regular lifestyle of women with children and the long observation period, the anthropometric data of the current study (current BMI, BMI 10 years ago, waist circumference, hip circumference, waist-hip ratio) did not differ between women with children and women without children. In contrast to our findings, the Cochrane review by Moran et al. [6] reported that patients with PCOS who had lifestyle interventions had significant weight loss (median loss $-3.47 \mathrm{~kg}, 95 \% \mathrm{CI}:-4.94$ to -2.00). Similarly, the waist-hip ratio also dropped significantly for patients who had lifestyle interventions (mean $-1.95 \mathrm{~cm}$; 95\% CI: -3.34 to -0.57 ).

In addition to excessive food consumption, another phenomenon which has occurred in industrialized nations over the last few decades is a reduction in sleep duration [12]. Epidemiological studies have shown a correlation between shorter sleep duration, weight gain and obesity, despite an increased energy expenditure of around $5 \%$ per 24 hours. Many parents, particularly in the early years of parenthood, are more sleep deprived than before, meaning that the negative impact of sleep deprivation on anthropometric parameters could negate the potentially beneficial effect of a more regular daily routine. Moreover, slightly fewer women with children reported exercising regularly compared to women without children, although the difference was not statistically significant (56 vs. $61.5 \%$ ).

One indication of the positive impact of a regular daily routine is the significant difference in prolactin levels as well as a difference in DHEAS levels which almost attained significance. Both hormones may increase with stress and were lower in women with children than in women without children. This is the first time a study focuses on fertility and its impact on subsequent, non-reproductive consequences of PCOS.

In the LIPCOS study testosterone and SHBG levels did not differ significantly between the group of women with children and the group of women without children and were within normal ranges for both groups. 
With regard to hyperandrogenism the meta-analysis of Moran and colleagues [6] showed a significantly higher decrease in serum testosterone levels in patients with PCOS who reported changes in lifestyle (mean difference [MD] - $0.27 \mathrm{nmol} / \mathrm{L}, 95 \% \mathrm{CI}$ : -0.46 to -0.09 ) but found no evidence for changes in sexual hormone-binding globulin (SHBG) or the free androgen index (FAI). Clinically, hirsutism (measured using the Ferriman Gallwey score) was reported to decrease in women who changed their lifestyle (mean $-1.19 ; 95 \% \mathrm{CI}$ : -2.35 to -0.03 ). It should be noted, however, that the period investigated by published studies was only between 12 and 48 weeks, which means that no study followed up the impact of lifestyle changes for longer than one year. SHBG levels are often decreased in patients with PCOS; levels are inversely correlated with body weight, BMI and waist-to-hip ratio (WHR). Energy intake and physical exercise influence SHBG production as do hormones, with estrogens and thyroxin increasing SHBG levels and insulin, IGF-1 and prolactin having the opposite effect. Diet also affects SHBG: a low carbohydrate diet lowers SHBG levels; a low protein and low fat diet increases SHBG [13]. In addition to hormonal status, nutrition could be responsible for the relatively normal SHBG levels in the patient population of this study.

The LIPCOS pilot study has certain limitations: the data on weight, cycle length, regular daily routine and nutrition are based on the information provided by the study participants and cannot be verified retrospectively. The prospective main study collected data on these issues objectively. Aspects such as "healthy diet" or "regular exercise" which study participants were asked about, are not precise quantitative terms. This can lead to subjective overestimation or underestimation of retrospectively reported changes, as retrospectively given information often has a tendency to be distorted. At the same time, the long period of observation and the semi-quantitative investigation of many important lifestyle aspects are advantages of this type of study approach. Questionnaires which are too detailed may limit the validity of a study because of the lower number of responses received, incomplete information provided, and low numbers of participants.

The prospective LIPCOS main study will - as soon as all study participants have been observed for 4 years - refine the hypotheses of the pilot study.

Another limitation of this study is that in the group of 39 women without children the study did not differentiate between those women who did not want to have a child and those who were unable to have a child. The possible causes of an inability to have children were not explored; this is a limitation as is the low numbers of participants whose data were available for analysis. More pronounced PCOS or stronger PCO characteristics could be the reason why some of the women who wanted children were unable to conceive. Because of the small number of women with children ( $n=25)$, this retrospective study did not differentiate between spontaneous and assisted reproduction. The fact that hormone investigations were not standardized with respect to the day of the menstrual cycle is also a limitation. If positive effects of having children on the long-term course of PCOS are confirmed over the further course of the LIPCOS study, systematic studies will be necessary to investigate these aspects in more detail.

Overall, the meta-analysis of Moran et al. confirmed the benefits of lifestyle changes in patients with PCOS. Because of the lack of data it was not possible to show an impact on fertility, but lower serum testosterone levels and reduced hirsutism were observed.
A lower risk for metabolic and cardiovascular events could not yet be demonstrated. In summary, the Cochrane review shows that randomized and controlled studies, in particular long-term studies, on the effect of lifestyle changes on women with PCOS are lacking, and more studies are needed. Valid data with regard to fertility and quality of life would be of particular interest. It would also be important that studies investigate differences between subgroups, for example, slim vs. obese women. Another challenge in this context is the new classification proposed by the 2012 NIH Experts Workshop which suggested differentiating between 4 phenotypes:

- Hyperandrogenemia and chronic anovulation

- Hyperandrogenemia and polycystic ovaries (with ovulatory cycles)

- Anovulation and polycystic ovaries (without hyperandrogenemia)

- Hyperandrogenemia, PCO and anovulation

There is a notion that women may move from one phenotype to another, but valid data on this point are still lacking. Investigating which medical or lifestyle interventions could be useful for which patients will be one of the challenges in coming years. There is a pressing need for more research before it is possible to speak of "targeted therapy" for patients with PCOS.

\section{Conclusion \\ $\nabla$}

The LIPCOS study showed for the first time that there may be an association between the course of PCOS and becoming pregnant - and vice versa. In this study women with children reported shorter menstrual cycles and had lower testosterone levels than women with no children.

\section{Conflict of Interest \\ $\nabla$}

None.

\section{References}

1 Conway G, Dewailly D, Diamanti-Kandarakis E et al.; ESE PCOS Special Interest Group. The polycystic ovary syndrome: a position statement from the European Society of Endocrinology. Eur J Endocrinol 2014; 171: $1-29$

2 Franks S, Stark J, Hardy K. Follicle dynamics and anovulation in polycystic ovary syndrome. Hum Reprod Update 2008; 14: 367-378

3 Dunaif $A$. Insulin resistance and the polycystic ovary syndrome: mechanism and implications for pathogenesis. Endocr Rev 1997; 18: 774800

4 Ehrmann DA. Polycystic ovary syndrome. N Engl J Med 2005; 352: 1223-1236

5 DeUgarte CM, Bartolucci AA, Azziz R. Prevalence of insulin resistance in the polycystic ovary syndrome using the homeostasis model assessment. Fertil Steril 2005; 83: 1454-1460

6 Moran LJ, Hutchison SK, Norman RJ et al. Lifestyle changes in women with polycystic ovary syndrome. Cochrane Database Syst Rev 2011; 7: CD007506

7 Fauser BJCM, Chang J, Aziz R et al. Revised 2003 consensus on diagnostic criteria and long-term health risks related to polycystic ovary syndrome (PCOS). Rotterdam ESHRE. Hum Reprod 2004; 19: 41-47

8 Geisthövel F, Botsch F, Wacker A et al. A novel classification of functional androgenization (FA) in women. Hum Reprod 2005; 20: 485

9 Sample WF, Lippe BM, Geyepes MT. Gray-scale ultrasonography of the normal female pelvis. Radiology 1977; 125: 477-483

10 Vigorito C, Giallauria F, Paloma $S$ et al. Beneficial effects of a threemonth structured exercise training program on cardiopulmonary functional capacity in young women with polycystic ovary syndrome. J Clin Endocrinol Metab 2007; 92: 1379-1384 
11 Legro RS. Impact of metformin, oral contraceptives, and lifestyle modification on polycystic ovary syndrome in obese adolescent women: do we need a new drug? J Clin Endocrinol Metab 2008; 93: 4218-4220

12 Chaput JP, St-Onge MP. Increased food intake by insufficient sleep in humans: are we jumping the gun on the hormonal explanation? Front Endocrinol (Lausanne) 2014; 5: 116
13 Thaler MA, Seifert-Klauss V, Luppa PB. The biomarker sex hormonebinding globulin - from established applications to emerging trends in clinical medicine. Best Pract Res Clin Endocrinol Metab 2015; DOI: http://dx.doi.org/10.1016/j.beem.2015.06.005 